\title{
Determinants and Possible Solutions of K12 Education Gap Between Chinese Urban and Rural Areas
}

\author{
Yijiao Jiang ${ }^{1, *, a, \dagger}$, You Wang ${ }^{2, *}$, b, $\dagger$ \\ ${ }^{1}$ Nanjing Normal University, 1 Wenyuan Road, Nanjing, t210023, China \\ ${ }^{2}$ University of Kansas, 1450 Jayhawk Blvd, Lawrence, KS 66049, United States \\ *Corresponding author.Email: ${ }^{*}$ s0359597@salemstate.edu, ${ }^{b} y w a n g 1104 @ k u . e d u$ \\ These authors contributed equally.
}

\begin{abstract}
Education is an important driving force for social development and human progress. Much work has been done to promote equity and development in education. The rapid development of the social economy has accelerated the pace of the development of education, but at the same time, the gap between urban and rural education has gradually increased. This paper mainly analyzes the reasons for the gap between urban and rural education in China:a) From the perspective of economic development, the unequal distribution of educational resources caused by the income gap between urban and rural areas has widened the gap between urban and rural education; b) From the dimension of cultural development, the gap between urban and rural cultural circles and traditional concepts affects the possibility of students in different areas to receive education, which also includes the reason for the cognition of gender inequality. To solve these problems, the article has provided several effective suggestions: a) Improve the quality of rural teachers, and pay attention to the most fundamental needs of rural teacher training; b) Set up the correct education concept, use the parent training, migrant workers return and other strategies to update the rural family education concept.
\end{abstract}

Keywords: Educational gap between Chinese urban and rural areas, $K-12$, teachers in rural areas, double reduction, China.

\section{INTRODUCTION}

In China, the differences in urban and rural development have led to the educational gap in Chinese urban and rural areas. Due to the different allocation of educational resources and financial conditions, the current situation between Chinese urban and rural areas is asymmetry. Specifically, in Chinese urban areas, with the popularization of compulsory education and the emphasis and investment on education, the popularization rate of education and the allocation level of teaching resources are quite high. In contrast, there is a huge gap between the education level of rural China and urban China. Specifically, the proportion of uneducated population in rural areas is much higher than that of the Chinese urban area, and the development of high-quality teachers and educational resources is lagging behind. Overall, Chinese rural children have no equal access to the educational resources and opportunities compared to urban children in terms of educational resources, thus bridging the gap between Chinese urban and rural areas is in urgent need.

In order to alleviate the educational gap between urban and rural areas in China, the K-12 education structure is feasible and necessary to achieve educational equity. As the short for kindergarten through 12 grade, K-12 refers to "the overall structure of education in the U.S. educational system from kindergarten to 12th grade" [1]. As an important group receiving basic education, K-12 can effectively alleviate the imbalance in the construction of educational facilities and the allocation of teacher resources between urban and rural areas in China to maximize educational equity. The existing literature shows that, "as a proven home education option, more than half of families in the United States have joined the K-12 education of new attempts" [2]. For example, when K-12 education is carried out in a rural area with relatively scarce resources in China, this system can provide personalized adaptive programs for learners at different stages 
through K-12 to improve learning efficiency and provide much educational resources for children in rural areas China. As a dominant part of education, K-12 is a system of jointly sharing resources to realize education, which plays a vital role in promoting educational equity and reducing the gap between Chinese urban and rural area. As a result, through the implementation of K-12, this educational framework can effectively solve the unequal distribution of educational resources, which is of great significance to the balance of high-quality educational resources and the coordinated integration of the educational development in Chinese urban and rural area.

The rapid development of the social economy has accelerated the pace of social development, but at the same time the education gap between urban and rural areas has gradually increased, which has a negative impact on the development of education in China. Consequently, we must pay attention to the gap between urban and rural education and take effective countermeasures to narrow the gap constantly and promote the good development of education in our country. In the existing literature, we can find a lot of research results on how urban and rural economic differences affect the education gap between urban and rural areas. Wang Jing, an education scholar at Lanzhou University of Commerce, argues that the different levels of economic development between urban and rural areas partly result in different educational opportunities for urban and rural populations. There are two reasons: the long-term existence of the policy of monopoly purchasing and marketing in history has caused the increasing poverty of farmers and the widening gap between urban and rural areas; The national investment in urban public service facilities is much higher than that in rural areas, coupled with the remote location, backward transportation, information occlusion and other natural conditions, which jointly aggravates the backward development of rural education [3]. However, the factors that cause the differences are far more than economic differences. In addition to the economic income gap, the distribution of teacher resources, the cognition of ideology (whether it is necessary for children to receive higher education), gender inequality and other factors also contribute to the education gap between urban and rural areas. Most of the methods given by the existing literature that are likely to narrow the gap between urban and rural education have been put into practice and achieved great results. But the gap still exists and needs to be narrowed. We need to study how to narrow the gap between urban and rural education in combination with the latest urban and rural status quo and the latest government policy trends.

Taking Chinese K-12 students as the research object, this paper mainly analyzes the reasons for the differences between urban and rural education. At the same time, this paper will also explore possible ways to narrow the gap between urban and rural education in the future and study the latest government policies, based on existing scientific research results and the latest status quo of urban and rural education in China.

\section{THE MAIN REASONS OF THE FORMATION OF GAP}

In China, the difference between urban and rural educational development are mainly determined by economic and cultural factors. In China, there are huge differences between urban and rural areas in terms of education investment, teaching quality, and teachers' level. Facing the serious imbalance of education development, it is important to adopt the K-12 system to promote education equity in China. Specifically, educational equity refers to "the state that citizens can freely share the current public education resources, including three different levels: the equity of educational opportunities, the equity of educational process, and the equity of educational results [4]." Considering the unequal distribution of urban and rural education and the economic and cultural differences, the fairness of K-12 education process, opportunities and resources become the key factors to solve the coordinated development of Chinese urban and rural area.

Primarily, in terms of economic aspects, the income gap between urban and rural areas determines the unbalanced distribution of educational resources in society. To some extent, income determines social status and access to more advantageous educational resources, and the distribution of educational infrastructure and resources is unbalanced in urban and rural areas. Due to the advantages of urban education in economic resources, good educational resources and teacher resources are concentrated in cities. Compared with the rural area, urban areas have unique advantages in infrastructure, educational resources, social wealth, talents and information. As Rawals states, "The essence of rural development and urban migration is the distribution of interests among people [5]." For example, due to the lack of employment opportunities in rural areas, parent migration to urban employment leads to left-behind children in rural areas, and the lack of funds and the loss of teacher resources in rural education result in a vicious circle. With the advancement of urbanization, the increasing number of rural migrant workers in cities has brought about the indifference of family culture atmosphere and the weakening of family education for the children of floating population. As Rhoda argues, "migration appears to be stimulated by interventions which increase access to cities, commercialize agriculture, strengthen rural-urban integration, raise education and skill levels [6]." In this case, children who are left at home in rural areas do not receive direct education from their parents, 
and the education provided by the elderly is inadequate for children. Thus, for rural areas, the education and development of left-behind children should be noticed and $\mathrm{K}-12$ is a good opportunity to promote the equity of educational resources.

In current, the discrepancy of economic structure in Chinese urban and rural area is becoming increasingly clear. In order to promote the rapid development of education, the state has implemented the policy of urban preference: increasing financial investment in urban basic education, which makes the allocation of rural basic education even more backward. To a certain degree, inadequate financial allocation of rural education and insufficient funds make the differences between urban and rural education more and more obvious. For instance, due to the increasing pressure of urban education competition, the middle class is increasingly competing for and investing in excellent educational resources. As a result, this predicament also leads to more educational resources and infrastructure flooding into cities, exacerbating the educational imbalance between rural and urban areas. Influenced by the focus of education development, education policies have gradually formed the value orientation of urban centers, that is, to satisfy or even reflect the interests of urban people first, thus resulting in a growing gap between urban and rural education. When it comes to equal educational opportunities, rural students are far behind their urban counterparts. Under the great pressure of urban education competition, the government mainly invests in urban education in the allocation of educational funds. In terms of teaching facilities and teaching quality, the quality of urban teachers is high, educational resources are relatively concentrated, and various educational facilities are relatively complete. As is illustrated by Monk, "the potential for modern telecommunication and computing technologies to offset some of the educational gap between urban and rural areas, thus affecting the equitable the educational development of these areas [7]". In the face of this situation, K-12 will play an extremely important role in adjusting the imbalance between urban and rural resource allocation and the adverse situation of lack of quality education resources in rural areas.

As for culture factors, the different endowments and characteristics of the cultural circles of urban and culture also have different effects educational views. Due to the difference in family background and concept between urban and rural areas, there is a huge gap between parents' awareness of their children's education in the two areas. As Theobald states, "the openness of urban culture makes it easier for cities to absorb society's mainstream social and cultural values, which brings more opportunities and opportunities for the development of schools and education [8]." For example, in rural areas, the preference for boys is more evident in education. Discussing the difference in views on education for girls and boys in rural Areas, Hannum put forward that "people living in rural Areas of China tend to live in a self-sufficient lifestyle, with little contact with the outside world and a relatively closed life [9]." In other words, rural parents believe that it is not necessary for girls to receive education. As a result, this feudal ideology also makes many rural women lose the opportunity to obtain further education, which exacerbates the difference between urban and rural education development.

Specifically, the difference between urban and rural educational development is closely related to the different cultural circles of Chinese urban and rural areas. Anderson and Judith explained the difference between rural and urban education, and pointed out that "in the core of rural culture, there is a traditional value concept which is relatively backward in urban areas, and it is characterized by rusticity, closure and singleness [10]." In China, rural areas are mainly engaged in agricultural production, which is distributed more dispersed than urban areas. To some extent, urban education is distinct from rural in the social norm to be contrasted with and some culture views to be measured or against. Facing this situation in terms of cultural difference, K-12 provides more opportunities for equal access to education in a relatively backward rural culture. In rural areas, the specific goal of educational equity is to achieve balanced allocation of educational resources and ensure the equity of children's right to education. Thus, in different cultural backgrounds of Chinese urban and rural area, the ultimate goal of educational equity through $\mathrm{K}-12$ can provide more possibilities to promote the balanced development opportunities for every student.

\section{SPECIFIC WAYS TO NARROW THE EDUCATIONAL GAP BETWEEN URBAN AND RURAL AREAS}

\subsection{Improve the quality of teachers in rural area}

From the perspective of education, teachers plays an essential role. They are the messengers of knowledge transmission. Improving the quality of teachers in rural areas is an urgent and difficult measure to achieve. When the difference of urban and rural education influence is no longer the hardware factors, teachers' quality and teaching skills level becomes extremely important, because we want to narrow the gap between urban and rural education is to the countryside student can stand on a higher level to contribute to the country at the same time also change the construction of their hometown and their life.

In recent years, the Chinese government has published a series of policies to enhance the quality of 
teachers in rural area. From the outline of the National Medium - and Long-term Education Reform and Development Plan (2010-2020) in 2010 [11] to the Guidance on Deepening the Training Mode for Primary and Secondary School Teachers and Comprehensively Improving the Quality of Training in 2013 [12] to the Rural Teacher Support Plan (2015-2020) in 2015 [13]. From "focusing on the rural primary and secondary school teachers" to "deepen the reform of primary and secondary school teachers training mode", to "according to the actual needs of teachers in rural areas to improve the way of training", reflects the state in order to improve the quality of rural primary and secondary school teachers for the overall train of thought, to improve the ability of teachers in rural areas quality as the key point, to improve rural teacher training mode for the gripper. However, in the current teacher training programs, the actual demands on rural teachers are masked. Failing to improve the training methods according to the actual needs of rural teachers will directly affect the training quality of rural teachers. Xiao Qinghua, a professor of education at Hangzhou Normal University, pointed out that the key to improving the training of rural teachers is to treat rural teachers as the core in the training, and to reflect and meet the core demands of rural teachers in the training [14]. How to develop the training strategies base on "core demands"? There are three ways. First, cultivate training objectives based on the professional development of rural teachers. Due to the heavy class burden of rural teachers, the training contents, methods, time and place can be flexibly sent to the countryside according to the actual needs of rural teachers, so as to send high-quality education and teaching training resources to rural schools. Through the professional support service team including backbone teachers, famous teachers, teaching and research personnel to carry out local training for rural teachers, combined with training resources inside and outside the school to promote rural teachers' professional development. Second, provide differentiated training contents for rural teachers. There are differences among rural teachers. Rural teachers in different school have different needs for professional development and training. Therefore, in order to reflect the demands of individual rural teachers in professional development, it is necessary to provide differentiated training content, try to ensure that every teacher can get professional sustainable development, and promote the differentiated development of teachers. For example, for rural primary school teachers, due to the relatively weak educational resources, government can mainly provide training of small group teaching, bilingual teaching (ethnic areas), rural culture, teaching organization skills, modern distance teaching and other aspects of training content; Finally, implement the training method of rural teacher flow system. To effectively meet the "core demands" of individual rural teachers in professional development, we should invite in high-quality education, teaching and training resources, and support rural teachers to go out and go to schools with better education and teaching resources on-the-spot training.

\subsection{Establish a correct idea of education}

In recent years, the government attaches more and more importance to education in rural area, increasing investment in the renovation of school buildings, adding hardware facilities, subsidizing the cost of accommodation and food, and creating a good environment for teachers and students [15]. In spite of this, a considerable number of parents try their best to send their children to urban schools, resulting in excessive class size in urban schools, shortage of educational resources and greater difficulty in management, while idle and wasted educational resources in rural schools. The government's investment is not in direct proportion to the effect. Student weariness phenomenon is serious, behaviour habit is bad, and moral idea is weak. The main reason for the above problems is that the cultural level of rural parents is low, some educational concepts are not correct, and some do not pay attention to the education of their children, to their children, that the children sent to school, it is the matter of the teacher whether to become successful [16]. Here are a few things we can do to change the status quo. First, parents' schools should be established. Relevant departments should supervise the development of parents' schools, so that parents' schools can play a vital role in school education. Family education and school education can go hand in hand, improving the educational concept of parents in rural areas. As a result, parents and children can grow up together. Second, the government should strengthen the development of rural areas and attract migrant workers to return to the countryside for career development. The backflow of migrant workers is beneficial to the renewal of the concept of family education in rural areas [17]. After migrant workers move to the economically developed areas, the lifestyle, educational concept and prosperous cultural scene of the developed areas will inevitably have different degrees of impact and influence on their ideas, which may change their concept and consciousness structure. From the demographic structure, the migrant workers are mainly young people, and they are easy to accept new ideas; Most of them have received compulsory education and have a certain sense of self-breakthrough. When those migrant workers return to the countryside, their new ideas and self-breakthrough consciousness will undoubtedly positively impact the renewal of family education concepts. For the revitalization of rural education, the renewal of the family education concept will be a favorable condition and opportunity. 


\subsection{The "Double Reduction" policy}

In July 2021, the Chinese government had recently announced an education policy called "double reduction"( Reducing the burden of homework and offcampus training for students in compulsory education). Compared with previous policies issued by local governments and the Ministry of Education, the "double reduction" policy has more in-depth and comprehensive educational guidance. The "double reduction" policy has also played a crucial role in narrowing the educational gap between urban and rural areas.

The "double reduction" policy mainly proposes the following reform directions: a) set clear requirements on the total amount and duration of homework: ensure that no written homework is assigned to grade one and grade two in primary school, but consolidation exercises can be arranged appropriately in school; The average writing time for grade 3 to 6 in primary school should not exceed 60 minutes, and that for junior high school should not exceed 90 minutes. b) Higher requirements are put forward for after-school service: in principle, after-school service should end no earlier than the local normal off-duty time; For students with special needs, schools should provide extended care services; Junior high school weekday evening can open self-study classes. We investigate and punish teachers who make up extra lessons for compensation outside school in accordance with laws and regulations, and even revoke their qualifications. c) The school shall ensure that students learn well in school: teaching shall be strictly based on the curriculum standards, and all teaching shall be done to ensure that students meet the academic quality standards stipulated by the state. Schools may not increase or reduce class hours, increase difficulty or speed up progress at will. d) Strict norms for off-campus training: no new off-campus training institutions of subject oriented to students in compulsory education will be approved in all regions, and the existing training institutions of subject oriented are uniformly registered as non-profit institutions. For the online discipline training institutions that were previously put on record, the examination and approval system will be changed. Disciplinary training institutions shall not be listed for financing and capitalization operation is strictly prohibited. It is strictly prohibited to exceed the standard and advance training, and non-discipline training institutions are strictly prohibited to engage in discipline training [18].

The "double reduction" policy is to effectively reduce the burden of students and parents, curb the spread and deterioration of "theater effect", reverse the myopic and utilitarian education concept, and build a healthy education ecology. Due to the income gap and other factors, parents in rural areas are unable to give their children more investment in extracurricular education in addition to compulsory education.
Implementing the "double reduction" policy helps rural students and urban students be in a relatively fair educational environment, receive the compulsory education in uniform standard (excluding extra tutoring), and make progress together.

\section{CONCLUSION}

In summary, this article mainly focuses on economic and cultural factors leading to education differences between urban and rural areas and possible solutions that might help solve the problem. We document that the income gap and cultural background determine that more advantageous educational resources are distributed unequally between urban and rural areas. The problems about left-behind children and education involution deserve society's attention. Also, the cultural factors involve parents' awareness of education and the lifestyles, which cause their different attitudes towards education between urban and rural areas.

To solve these problems, the current article has provided several effective suggestions. In the first place, it's necessary to improve the quality of rural teachers. Some special teacher training programs are in the process of incubating more high-quality teachers now. Also, it's important to establish a correct idea of education. The government has invested a lot in providing more ideal education environment for rural areas. These policies are formulated to narrow the income gap and promote education equality in recent years.

Although this article points out some core problems of education in urban and rural areas and mentions a few useful solutions, it also has several limitations. There are always other determinants of education inequality for researchers to search and more changeable situations to consider. This article is believed to inform and motivate future research in these areas, which will help create a brighter future of education

\section{REFERENCES}

[1] M.K. Barbour, K. Kennedy. K-12 online learning: A worldwide perspective. Education Faculty Publications, 18(2), 2014, 6-12.

[2] G.S. Demetrios, (2017). Investigating the Potential of the Flipped Classroom Model in K-12 ICT Teaching and Learning: An Action Research Study. Journal of Educational Technology \& Society, 20(1):261-273.

[3] J.Wang,\& Evergreen College of Lanzhou University of Commerce. (2014). The impact of urban-rural education gap on urban-rural income gap in China. Intelligence. Published. 
[4] P. Bourdieu. (1997). "Cultural Reproduction and Social Reproduction”. In : Power and Ideology on Education. Oxford Press, 1997.

[5] J.Rawls, (2000). A Theory of Justice( Revised Edition). Belknap \& Harvard University Press.

[6] P.M. Anderson, \& S.P. Judith, (2010). Why Is Urban Education Different from Suburban and Rural Education? Counterpoints, Vol. 215, No,19, pp. 273-283.

[7] E. Hannum, (2003). Poverty and Basic Education in Rural China: Villages, Households, and Girls' and Boys' Enrollment. Comparative Education Review, Vol. 47, No. 2 , pp. 141-159.

[8] D.H. Monk, (2007). Recruiting and Retaining HighQuality Teachers in Rural Areas. The Future of Children, Vol. 17, No. 1, pp. 155-174.

[9] R. Rhoda, (1983). Rural Development and Urban Migration: Can We Keep Them down on the Farm? The International Migration Review, Vol. 17, No. 1, pp. 34-64.

[10] P. Theobal, (2005). Urban and Rural Schools: Overcoming Lingering Obstacles. The Phi Delta Kappan, Vol. 87, No. 2, pp. 116-122.

[11] Ministry of Education of China. (2013, 5 8). The Guidance on Deepening the Training Mode for Primary and Secondary School Teachers and Comprehensively Improving the Quality of Training. Retrieved from Ministry of Education of the People's Republic of China: http://www.moe.gov.cn/srcsite/A10/s7034/201305/ t20130508_151910.html

[12] Ministry of Education of China. (2015, 6 1). Rural Teacher Support Plan (2015-2020). Retrieved from Ministry of Education of the People's Republic of China:

http://www.moe.gov.cn/jyb_xxgk/moe_1777/moe_ 1778/201506/t20150612_190354.html
[13] Ministry of Education of China. (2010, 7 29). The outline of the National Medium - and Long-term Education Reform and Development Plan. Retrieved from The Central People's Government of the People's Republic of China: http://www.gov.cn/jrzg/201007/29/content_1667143.htm

[14] Ministry of Education of China. Bureau of finance of China. National Development and Reform Commission. (2021, 6 23). Opinions on deepening the improvement of weak links in compulsory education and enhancing its capacity. Retrieved from Central People's Government of the People's Republic of China: http://www.gov.cn/zhengce/zhengceku/202107/03/content_5622097.htm

[15] Q. Xiao, (2020). The Discussion Based on "Core Demands" of the Training of Teachers in Rural Areas. Journal of The Chinese Society of Education, 8, 93-96.

[16] W. Yi, (2013). Suggestions on attaching importance to rural education and promoting balanced development of urban and rural education. Haikou: The People's Government of Hainan Province.

[17] S. Junyang, (2021). Opportunities, challenges and strategies for the development of rural education in western China during the 14th Five-year Plan period. Ethnic Education of China, 1, 23.

[18] Chinese General Office of the State Council. Suggestions on further reducing the burden of homework and off-campus training for students in compulsory education. (2021.7) Retrieved from Central People's Government of the People's Republic of China: http://www.gov.cn/zhengce/202107/24/content_5627132.htm 